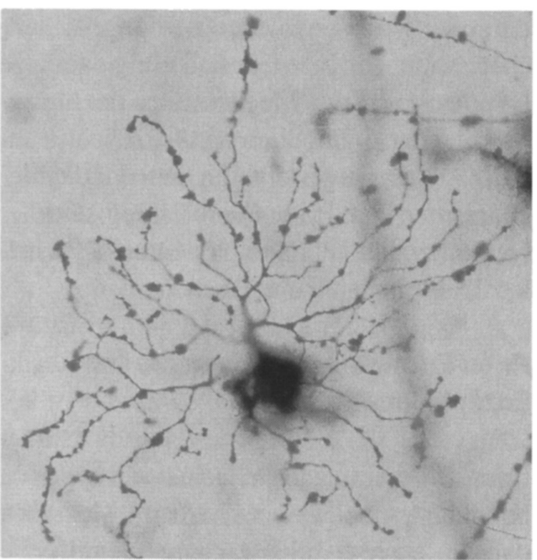

Titelbild:

Bistratifizierte Ganglienzelle aus der Retina des Kaninchens, Golgi-Cox-Färbung;

Durchmesser des Dendritenfeldes ca. 200 $\mu \mathrm{m}$. Siehe den Artikel von Ernst-August Seyfarth und Leo Peichl (Präparat: Brian B. Boycott) ab Seite 34

HAUPTARTIKEL

Adriano Aguzzi und Sebastian Brandner

Molekulare Mechanismen der Prion-Toxizität

Siegfried Kösel, Rupert Egensperger, Christoph B. Lücking, Nina M. Schnopp, Parviz Mehraein und Manuel B. Graeber

Genotyp-Analyse bei Hirnerkrankungen

\title{
Thomas Knöpfel
}

Metabotrope Glutamatrezeptoren -

Multidisziplinäre Grundlagenforschung mit Bedeutung für die Medizin

TAGUNGSBERICHTE

\section{Gerald Seifert und Christian Steinhäuser}

2. Europäische Tagung: „Glial Cell Function in Health and Disease“

FORSCHUNGSFÖRDERUNG

\section{Diethelm Richter}

SFB 406: Synaptische Interaktionen in neuronalen Zellverbänden

Neue neurowissenschaftliche Förderschwerpunkte in der DFG

INTERNET

\section{Ulrich Dirnagl}

Neurowissenschaften im World Wide Web

Prof. Dr. Michael Frotscher

1. Vorsitzender

Prof. Dr. Norbert Elsner

Stellvertretender Vorsitzender

Prof. Dr. Hans-Joachim Pflüger

Geschäftsführer

Dr. Helmut Kettenmann

Schriftührer

Prof. Dr. Rolf Heumann

Sektionssprecher

Molekulare Neurobiologie

Prof. Dr. Hans-Joachim Freund

Sektionssprecher

Klinische Neurowissenschaften

Prof. Dr. Klaus-Peter Hoffmann

Sektionssprecher Systemneurobiologie

Dr. Christian M. Müller

Sektionssprecher Zelluläre

Neurobiologie

Prof. Dr. Hans-Ulrich Schnitzler

Sektionssprecher

Verhaltensneurobiologie •

Prof. Dr. Claudia Stuermer

Sektionssprecherin Entwicklungs-

neurobiologie und Neurogenetik

Historisches Portrait

\section{Ernst-August Seyfarth und Leo Peichl}

„Wie Tuschezeichnungen auf Japanpapier“ -

vor 90 Jahren erhielten Golgi und Cajal den Nobelpreis

Nachrichten aus der Neurowissenschaftlichen Gesellschaft

Protokoll der Mitgliederversammlung

Methodenkurs: Methoden in der Neuropathologie

Methodenkurs: Optische Untersuchungsverfahren zur Messung von

Blutfluß, Metabolismus und Funktion des Gehirns

Mitgliederverzeichnis des Arbeitskreises Neuropharmakologie und -toxikologie

Neue Sektion Neuropharmakologie und -toxikologie?

Termine

Produkte 